\title{
Review: several drugs, especially triptans, are effective for pain relief in acute migraine
}

\author{
Oldman AD, Smith LA, McQuay HJ, Moore A. Pharmacological treatments for acute migraine: quantitative systematic
} review. Pain. 2002 Jun;97:247-57.

\section{QUESTION: In patients with acute migraine, are pharmacological treatments better than placebo for short and long term pain relief?}

Sources of funding:

Pain Relief Funds and

Pfizer UK, Ltd.

For correspondence:

Dr R A Moore, Oxford

Oxford, UK. Email

andrew.moore@

pru.ox.ac.uk.
Radcliffe Hospital,

\section{Data sources}

Studies in any language were identified by searching Medline (1966-2000), EMBASE/Excerpta Medica (1980-2000), the Cochrane Library, and the Oxford Pain Relief Database. Pfizer Inc. was contacted for data on all randomised controlled trials (RCTs) of eletriptan.

Pharmacological treatment $v$ placebo for acute migraine (range)*

\begin{tabular}{lllll}
\hline Outcomes & Treatment & Placebo & RBI & NNT \\
Headache relief at $2 \mathrm{~h}$ & $34 \%$ to $79 \%$ & $22 \%$ to $35 \%$ & $150 \%$ to $280 \%$ & 2 to 6 \\
\hline Headache relief at $1 \mathrm{~h}$ & $14 \%$ to $70 \%$ & $8.9 \%$ to $26 \%$ & $110 \%$ to $320 \%$ & 3 to 11 \\
\hline Pain free at $2 \mathrm{~h}$ & $11 \%$ to $60 \%$ & $4.7 \%$ to $12 \%$ & $240 \%$ to $640 \%$ & 3 to 9 \\
\hline Sustained relief at $24 \mathrm{~h}$ & $35 \%$ to $53 \%$ & $14 \%$ to $27 \%$ & $150 \%$ to $300 \%$ & 4 to 9 \\
\hline${ }^{*}$ Abbreviations defined in glossary. & & &
\end{tabular}

\section{COMMENTARY}

Which of the many drugs available for treatment of acute migraine should a busy clinician use? In the absence of a large, government-funded, super trial comparing all currently available treatments, we must synthesise information from other sources to assess their relative merits. The conclusions of the systematic review by Oldman et al are broadly consistent with head to head trial results, clinical experience, and the results of another systematic review ${ }^{1}$ : Firstly, triptans as a class outperform such non-specific medications as non-steroidal anti-inflammatory drugs; secondly, subcutaneous sumatriptan is the most effective treatment for acute migraine; and thirdly, with the important exception of naratriptan, which is less effective than the others, available oral triptans have similar efficacy. Rank orderings of these drugs based on the number needed to treat or other derived variables have limited clinical relevance because differences are small and confidence intervals overlap. Comparative inferences are also limited because the data do not represent head to head RCTs and placebo responses vary between trials, indicating differences in study populations.

Through no fault of its authors, this review provides no information about several important issues in abortive treatment of migraine. No trials of such treatments as narcotics met the quality standards for inclusion in this analysis, so information enabling us to put these drugs in context is lacking. This review also did not identify results for barbiturate-containing compounds (eg, Fiorinal [butalbital, aspirin, and caffeine], Novartis) that are commonly prescribed in the United States or 2 other triptans (almotriptan and frovatriptan) that are available. Trial results suggest that frovatriptan, ${ }^{2}$ like naratriptan, is substantially less effective than other triptans at the 2-hour efficacy end points recommended by the International Headache Society. ${ }^{3}$ Finally, the poor quality of adverse event reporting in clinical trials, as commented upon by the authors, is disappointing. Patients and physicians commonly base treatment decisions on a combined assessment of efficacy and tolerability, and the information needed to put this element of decision-making in perspective is lacking.

Elizabeth Loder, MD

Harvard Medical School, Boston, Massachusetts, USA

1 Ferrari MD, Roon KI, Lipton RB, et al. Oral triptans (serotonin 5-HT (1B/1D) agonists) in acute migraine treatment: a meta-analysis of 53 trials. Lancet 2001;358:1668-75.

Ryan R, Geraud G, Goldstein J, et al. Clinical efficacy of frovatriptan: placebo-controlled

3 studies. Headache 2002;42 (Suppl 2) 284-92. International Headache Society Committee on Clinical Trials in Migraine. Guidelines for controlled trials of drugs in migraine. IHS members' handbook. Oslo: Scandinavian Uni-
versity Press; 1997 .

\section{Study selection}

Studies were selected if they were double-blind, placebocontrolled RCTs of pharmacological treatment for acute migraine (International Headache Society diagnostic criteria for migraine with or without aura) in adults. Other inclusion criteria were single migraine attack, single-dose treatment at standard doses, baseline pain of moderate or severe intensity, and dichotomous or percentage data for $\geq 1$ efficacy outcome.

\section{Data extraction}

Studies were read independently by 2 reviewers and assigned a quality score. Data were extracted on study design, number of patients, dosing regimens, and timing or type of rescue medication. Outcomes were headache relief at 1 and 2 hours, freedom from pain at 2 hours, sustained relief for 24 hours, freedom from pain for 24 hours, and adverse effects within 24 hours after treatment.

\section{Main results}

48 reports of 54 trials were included (median size of treatment groups 126 patients). For headache relief at 1 and 2 hours, information was available for 13 oral interventions (11 for $1 \mathrm{~h}$ ), 2 intranasal interventions, and subcutaneous sumatriptan. All but 1 intervention (Cafergot [ergotamine tartrate plus caffeine], Novartis) was more effective than placebo. For freedom from pain at 2 hours, information was available for 13 oral interventions, intranasal sumatriptan, and subcutaneous sumatriptan. All interventions were better than placebo except for Cafergot. For sustained pain relief for 24 hours, information was available for 8 oral interventions and subcutaneous sumatriptan. All interventions were better than placebo except for zolmitriptan, $5 \mathrm{mg}$. Triptans seemed to be the most effective drugs, but no drug to drug comparisons were reviewed. No trials reported freedom from pain for 24 hours. Side effects could not be analysed because data were collected for a 7 to 10 day period and could not be separated from contamination with rescue medication or second doses.

\section{Conclusions}

Most pharmacological treatments, except Cafergot, are effective for pain relief in patients with acute migraine. Subcutaneous sumatriptan and newer oral triptans seem to be the most effective drugs for aborting migraine attacks, but drug to drug comparisons have not been done. Data on adverse effects are lacking. 